\title{
Tungsten and Molybdenum Surfaces Exposed to Warm Deuterium Ion Plasma with $Q$-Nonextensive Distribution of Electrons
}

S. Basnet and R. Khanal

Journal of Nepal Physical Society

Volume 6, Issue 1, June 2020

ISSN: 2392-473X (Print), 2738-9537 (Online)

Editors:

Dr. Binod Adhikari

Dr. Manoj Kumar Yadav

Mr. Kiran Pudasainee

JNPS, 6 (1), 50-58 (2020)

DOI: http://doi.org/10.3126/jnphyssoc.v6i1.30517

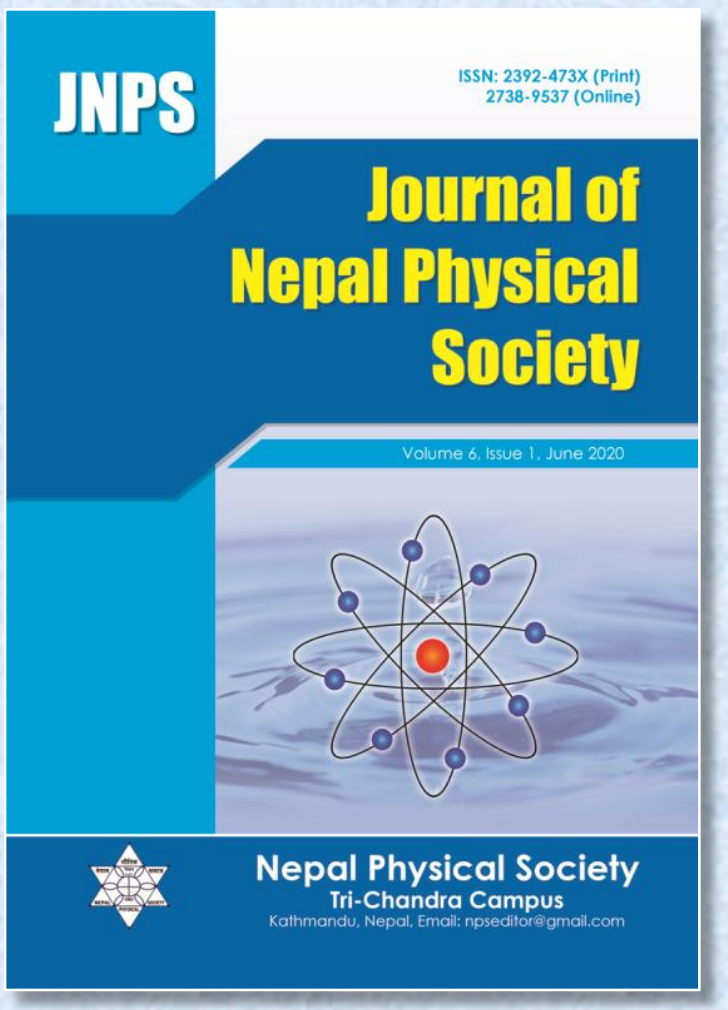

Published by:

Nepal Physical Society

P.O. Box: 2934

Tri-Chandra Campus

Kathmandu, Nepal

Email:npseditor@gmail.com 


\title{
Tungsten and Molybdenum Surfaces Exposed to Warm Deuterium Ion Plasma with $Q$-Nonextensive Distribution of Electrons
}

\author{
S. Basnet* and R. Khanal \\ Central Department of Physics, Tribhuvan University, Kirtipur, Kathmandu 44613, Nepal \\ *Corresponding Email: sbplasma1986@gmail.com, sbasnet@tucdp.edu.np
}

Received: 16 Jan., 2020; Revised: 18 May, 2020; Accepted: 28 Jun., 2020

\begin{abstract}
This work is concerned with the effect of non-Maxwellian electrons and obliqueness of magnetic field on magnetized plasma sheath characteristics, in which plasma interacts with tungsten (W) and molybdenum (Mo) surfaces via non-neutral plasma sheath using two fluids model. It is assumed that the singly charged positive ions are treated as warm fluid whereas the electrons obey $q$-nonextensive distribution. It is found that the $q$-nonextensive distributed electrons and the temperature of ions affect the entrance velocity of positive ions, which is a key parameter in the plasma sheath formation. Also, the nonextensive parameter $q$ affects the distribution of ions and electrons in the sheath region and their distributions explicitly related with the electrostatic potential variation. The parallel and perpendicular components of ions velocity are affected by the obliqueness of magnetic field. As the nonextensivity of electrons increases, the gradient in electric potential increases towards the wall and hence the impact energy also increases. The obliqueness of magnetic field and impact energy of ions is a key factor that determines the physical sputtering rate, particle reflection and absorption from the target surface. Furthermore, the probability of particle reflection coefficient from the $\mathrm{W}$-surface is higher than that of Mo-surface.
\end{abstract}

Keywords: Particle absorption, Particle reflection, Plasma facing materials, Plasma sheath, Sputtering.

\section{INTRODUCTION}

The study of plasma-wall transition mechanism which exists between quasineutral plasma and boundary surface is one of the interesting research fields in plasma physics $[1,2]$. During the past several years, there are many literatures on plasma sheath and presheath characteristics due to its wide range of growing applications or even growing interest in diverse fields [3-5]. The investigation of plasma sheath is a difficult task as it exhibits complex structure in the presence or absence of magnetic field. When the plasma comes in contact with material surface, plasma interacts with the surface via nonneutral plasma sheath formed at the vicinity of material wall. The plasma sheath characteristics has crucial role in the flow particles and energy towards the wall. The incident particle fluxes and energy transmits through the sheath towards the wall determine the life time of plasma facing materials (PFMs) [6]. For example, the first material wall in the fusion devices is exposed with high energetic particle flux $\left(\approx 10^{24} \mathrm{~m}^{-2} \mathrm{~s}^{-1}\right)$ at energies ranging from $\mathrm{eV}$ to $\mathrm{keV}$ which results to affect the life span of PFMs [7, 8]. Therefore, the proper design of divertor wall and material selection is very important. The understanding of plasma sheath properties in the presence as well as in the absence of magnetic field is fundamental for various plasma applications such as in divertor wall of fusion devices, surface modifications of materials, sputtering, etching and many more [9-11].

Tungsten (W) is the leading high- $\mathrm{Z}$ candidate surface material for future fusion devices and the first wall as a plasma facing components (PFCs) [12]; however, the high- $\mathrm{Z}$ material molybdenum (Mo) is also considered as an alternate to tungsten [13]. The reason behind considering high-Z materials as plasma facing components (PFCs) are high melting point, good thermo-mechanical properties and low physical sputtering rate of such materials [14].

In the past few decades, the nonextensive statistical mechanics has received a considerable interest in the plasma and its applications. It has been successfully applied to describe the interesting 
problems associated to astrophysics and space physics $[15,16]$. Tsallis proposed a concept of $q$ nonextensive entropy as a generalized form of the Boltzmann-Gibbs free entropy [17, 18]. Mathematically, Tsallis entropy can be written as;

$$
S_{q}=k_{B} \frac{1-\sum_{k=1}^{w}\left(p_{k}\right)^{q}}{q-1}
$$

in which $k_{B}$ is Boltzmann constant, $p_{k}$ is the probability of $k^{\text {th }}$ microstate, $w$ is the total number of microstates and $q$ is the degree of nonextensivity of the thermodynamic system. As the nonextensive parameter tends to unity i.e., $q \rightarrow 1$, equation (1) reduces to the usual Boltzmann-Gibbs entropy as;

$$
S_{q}=-k_{B} \sum_{k} p_{k} \ln p_{k}
$$

Pioneering work [19] gave an empirical formula for the particle reflection and energy reflection coefficients. The work was primarily focused on particle reflection and absorption coefficients from the target surface due to lighter ions (such as hydrogen, deuterium and helium) with the energy range from $10 \mathrm{eV}$ to $100 \mathrm{keV}$. This energy range encompassed the situations of fusion devices. It was found that the reflection and absorption of particles from the target surface not only depend on the kinetic energy of projectiles, but also on the mass ratio of target to projectiles.

In this work, we are interested to understand the plasma-wall interaction mechanism when the warm deuterium ion plasma interacts with the tungsten and molybdenum surfaces in the presence of $q$ nonextensive distributed electrons. The nonextensive distributed electron has considerable effect on particles and energy flow towards the wall. The impact energy with which the positive ions strike the target surface determines the physical sputtering process, particle reflection and absorption coefficients which are systematically presented in this work. The obtained results have useful applications such as in modeling of divertor wall in fusion device.

\section{DESCRIPTION OF MODEL AND BASIC EQUATIONS}

The electropositive magnetized plasma consists of singly charged positive deuterium ions and electrons. In the present work, we consider a model with onedimension in spatial coordinate and three dimension in velocity coordinates $v_{i x}, v_{i y}$ and $v_{i z}$. Also, the collision mean free path between ion and neutral is longer than the dimension of considered model. Thus, it is considered that there are no collisions in the plasma sheath region i.e., plasma sheath is collisionless. The magnetized plasma is bounded by two planar surfaces at $x=0$ (left hand boundary i.e., particle injecting side or sheath entrance) and $x=x_{\mathrm{w}}$ (right hand boundary or material wall) as shown in Fig. 1. A uniform magnetic field $\widetilde{B}$ acts in the xzplane, makes an angle $\psi$ with the normal to the wall which is also the direction of electric field. The plasma is non-isothermal i.e., $T_{e} \neq T_{i}$, where $T_{e}$ and $T_{i}$ are electron and ion temperatures, respectively. In the presence of oblique magnetic field, the velocity has two components:

$v_{\|}=v_{i x} \cos \psi+v_{i z} \sin \psi$

is the velocity parallel to the magnetic field which provides the forward movement to the ions and

$v_{\perp}=\left[v_{i y}^{2}+\left(v_{i z} \cos \psi-v_{i x} \sin \psi\right)^{2}\right]^{0.5}$

is the velocity perpendicular to the magnetic field which provides the rotational motion to the particles around the magnetic field [20].

As the electrons follow the Tsallis statistical mechanics, the velocity distribution function is deviated from the Maxwellian distribution. The $q$ nonextensive velocity distribution function for electrons is [21]

$f_{e}(x, \hat{v})=A_{q}\left[1-(q-1)\left\{\frac{E_{k}}{k_{B} T_{e}}-\frac{e \phi}{k_{B} T_{e}}\right\}^{1 / q-1}\right.$

in which $A_{q}$ is the normalization constant and $E_{k}$ is the kinetic energy of electrons.

The electron density distribution is obtained as;

$n_{e}(\phi)=\iiint f_{e}(x, \rho) d^{3} v$

which yields,

$n_{e}(\phi)=n_{e 0}\left[1+(q-1) \frac{e \phi}{k_{B} T_{e}}\right]^{\frac{3 q-1}{2 q-2}}$

in which $n_{e}, e$ and $\phi$ are electron density at the particle injection side $(x=0)$, electronic charge and electrostatic electric potential, respectively. 


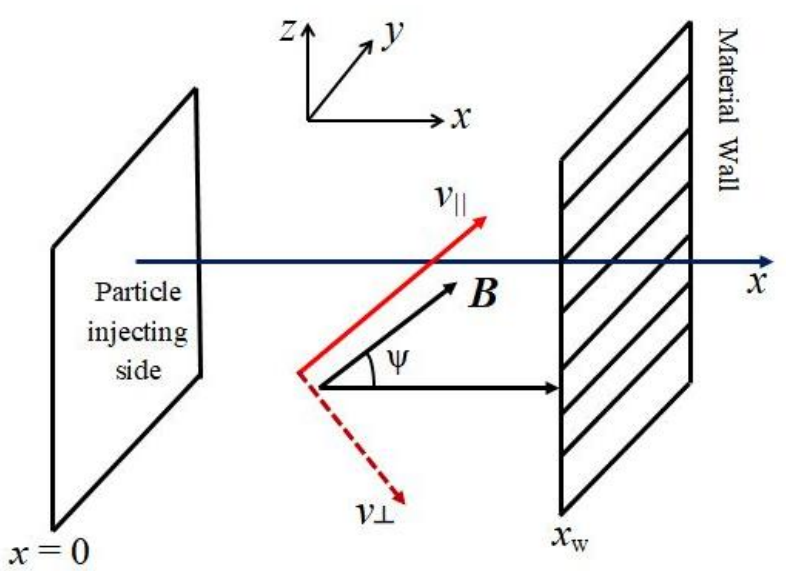

Fig. 1: Schematic geometry of magnetized plasma exposed with material wall.

In the absence of source, the steady state onedimensional continuity equation is written as;

$\frac{d}{d x}\left(n_{i} v_{i x}\right)=0$

and the momentum transport equation for ion fluid is

$m_{i} n_{i}\left(\nabla \cdot \rho_{i}\right) \rho_{i}=-e n_{i} \nabla \phi+e n_{i}\left(\rho_{i} \times \breve{B}\right)-\nabla p_{i}$

where $m_{i}, n_{i}$ and $v_{i}$ are the mass, density and velocity of the positive ions, respectively.

The one-dimensional Poisson's equation connecting electrostatic potential and charge density can be written as;

$\varepsilon_{0} \frac{d^{2} \phi}{d x^{2}}=-e\left(n_{i}-n_{e}\right)$

where $\varepsilon_{0}$ is the electric permittivity of free space.

The plasma is quasineutral at the sheath entrance and charge neutrality condition can be written as;

$n_{i 0} e-n_{e 0} e=0$

To solve the compiled set of fluid equations numerically, we introduce the transformations as;

$\xi=x / \lambda_{D e}, \quad \eta=-e \phi / k_{B} T_{e}, \quad u_{i}=\nu_{i} / C_{s}, \quad N_{i}=n_{i} / n_{e 0}$

$N_{e}=n_{e} / n_{e 0}, \Theta=T_{i} / T_{e}$, where $\lambda_{D e}=\sqrt{\varepsilon_{0} k_{B} T_{e} / n_{e 0} e^{2}}$ is

the electron Debye length and $C_{s}=\sqrt{k_{B} T_{e} / m_{i}}$ is the ion acoustic velocity.

Therefore, equations (7) - (10) becomes

$$
\begin{aligned}
& N_{e}=[1-(q-1) \eta]^{\frac{3 q-1}{2 q-2}} \\
& N_{i} u_{i x}=u_{i x}(\xi=0) \\
& {\left[u_{i x}-\frac{\Theta}{u_{i x}}\right] \frac{d u_{i x}}{d \xi}=\frac{d \eta}{d \xi}+\omega u_{i y} \sin \psi} \\
& u_{i x} \frac{d u_{i y}}{d \xi}=\omega\left(u_{i z} \cos \psi-u_{i x} \sin \psi\right) \\
& u_{i x} \frac{d u_{i z}}{d \xi}=-\omega u_{i y} \cos \psi \\
& \frac{d^{2} \eta}{d \xi^{2}}=\frac{u_{i x}(\xi=0)}{u_{i x}}-[1-(q-1) \eta]^{\frac{3 q-1}{2 q-2}}
\end{aligned}
$$

where $u_{i x}(\xi=0)=v_{i x}(x=0) / C_{s} \quad$ is the ion's velocity at the sheath entrance or at particle injection plane, $\omega_{i c}=e B / m_{i}$ is the ion cyclotron frequency and $\omega=\omega_{i c} \lambda_{D e} / C_{s}$ is the normalized frequency parameter.

The Bohm sheath criterion requires that ions must enter the sheath region with a velocity greater than the ion acoustic velocity. The Bohm sheath condition can be written as

$\left[\frac{d n_{i}}{d \phi}-\frac{d n_{e}}{d \phi}\right]_{\phi \rightarrow 0} \leq 0$

which yields,

$u_{i x}(\xi=0) \geq \sqrt{\Theta+\frac{2}{3 q-1}\left(1+\frac{\omega u_{i y 0} \sin \psi}{\frac{d \eta(\xi=0)}{d \xi}}\right)}$

This equation (19) is the modified Bohm sheath condition for magnetized plasma in the presence of thermal ions and $q$-nonextensive distributed electrons.

\section{WALL SPUTTERING YIELD, PARTICLE REFLECTION AND ABSORPTION COEFFICIENTS}

In the sheath region, the electric field is so strong that the motion of charged particles is governed by the electric field rather than magnetic field. The positive ions are accelerated by the electric field and most of the ions incident normally at the wall [20]. 
The nuclear stopping power based on the $\mathrm{Kr}-\mathrm{C}$ interaction potential is written as [22]

$$
S_{n}\left(\varepsilon_{T}\right)=\frac{0.5 \ln \left(1+1.2288 \varepsilon_{T}\right)}{\varepsilon_{T}+0.1728 \varepsilon_{T}^{0.5}+0.008 \varepsilon_{T}^{0.1504}} .
$$

with the reduced energy is defined as

$$
\varepsilon_{T}=\frac{m_{t}}{m_{i}+m_{t}} \frac{a_{L}}{Z_{i} Z_{t} e^{2}} E_{0}
$$

in which $m_{t}$ and $Z_{t}$ are the mass and charge state of the target particles, respectively, $m_{i}$ and $Z_{i}$ are the mass and charge state of the incident particles, respectively.

The impact energy of the incident particle $(\mathrm{eV})$ at the wall is calculated as;

$$
E_{0}=\frac{1}{2 e} m_{i} v_{i w}^{2}
$$

The parameter $a_{L}$ is the Lindhard screening length given by

$$
a_{L}=\left(\frac{9 \pi^{2}}{128}\right)^{1 / 3} \frac{a_{0}}{\left(Z_{i}^{2 / 3}+Z_{t}^{2 / 3}\right)^{0.5}}
$$

where $a_{0}$ is the Bohr's atomic radius.

The empirical relation for the calculation of physical sputtering yield for the normal incidence of projectile on the wall is [22];

$$
Y\left(E_{0}\right)=Q S_{n}\left(\varepsilon_{T}\right)\left[1-\left(\frac{E_{t h}}{E_{0}}\right)^{2 / 3}\right]\left(1-\frac{E_{t h}}{E_{0}}\right)^{2}
$$

in which $E_{t h}$ is the threshold energy and $Q$ is the fitting parameters. Below the threshold energy value, the equation (24) gives the unpredicted yields and this empirical relation of sputtering yield is modified as [22];

$$
Y\left(E_{0}\right)=Q S_{n}\left(\varepsilon_{T}\right) \frac{\left(\frac{E_{0}}{E_{t h}}-1\right)^{\mu}}{\lambda+\left(\frac{E_{0}}{E_{t h}}-1\right)^{\mu}}
$$

where $\lambda$ and $\mu$ are fitting parameters.
For normal incidence of lighter particle, the empirical formula for the particle reflection coefficient is [19]

$$
R_{N}=\frac{a_{1} \ln \left(a_{2} \varepsilon_{T}+2.718\right)}{1+a_{3}\left(\varepsilon_{T}\right)^{a_{4}}+a_{5}\left(\varepsilon_{T}\right)^{a_{6}}}
$$

where $a_{1}$ to $a_{6}$ are the constants that depends on the mass ratio of target to incident particles.

The fraction of particles absorbed $\left(R_{A}\right)$ at the material surface is given by

$R_{N}+R_{A}=1$

To obtained the more stable numerical fluid solutions, the initial conditions considered at the particle injection side are; $\eta(\xi=0)=0$, $d \eta(\xi=0) / d \xi=0.01$ and $u_{i}=\left(u_{i x 0}, u_{i y 0}, 0\right)$. It is assumed that the electrostatic potential is zero at the sheath entrance; however, it has taken a small residual electric field at the sheath entrance in order to prevent the divergence of numerical results. In addition, the presence of small electric field at the sheath entrance ensures the validity of plasma approximation. This non-zero electric field at the sheath entrance that acts along the $\mathrm{x}$-direction and the oblique magnetic field $\vec{B}$ on the xz-plane results in a drift in the y-direction as

$u_{i y 0}=-\frac{d \eta(\xi=0) / d \xi}{\omega} \sin \psi$

In this work, the physical parameters considered are; $n_{e 0}=10^{19} \mathrm{~m}^{-3}, B=5 \mathrm{~T}, \psi=(1-30)$ Deg., $T_{e}=$ $50 \mathrm{eV}$, and $T_{i}=20 \mathrm{eV}$.

\section{RESULTS AND DISCUSSION}

To study the plasma-wall interaction mechanism, first of all we have solved the set of normalized fluid equations (12)-(17) along with equation (19) and calculate the velocity of ions reaching the wall $\left(v_{i w}\right)$. Further, we have solved the equations (20)(27) to study the sputtering yield, particle reflection and absorption coefficients.

The velocity variation of positive ions at the sheath entrance is a key parameter in the plasma sheath formation close to the wall. The effect of thermal motion of ions and nonextensive distributed electrons on entrance velocity of positive ions is shown on Fig. 2. It is found that the velocity of ions at the sheath entrance increases with the increase in 


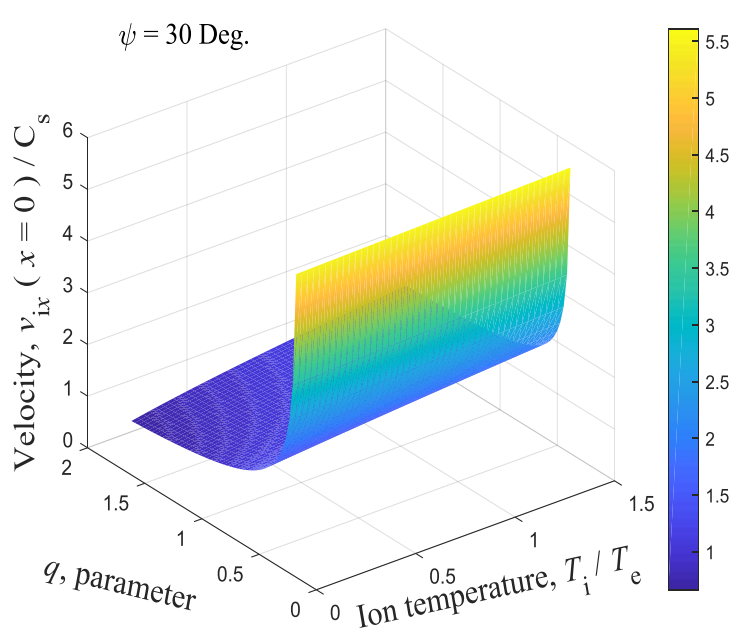

Fig. 2: Variation of ion velocity at the sheath entrance as the function of ion temperature and nonextensive parameter.

ion temperature and decreases with the increase in nonextensive parameter $q$. The marginal Bohm sheath condition $u_{i x}(x=0)=1$ is obtained when the nonextensive distributed electrons becomes Boltzmann distribution and ion temperature $T_{i}=$ $0.4 T_{e}$. The presence of $q$-nonextensive distributed electrons affect the variation of ion and electron density profiles in the plasma sheath region which are displayed on Fig.3 and Fig.4, respectively.

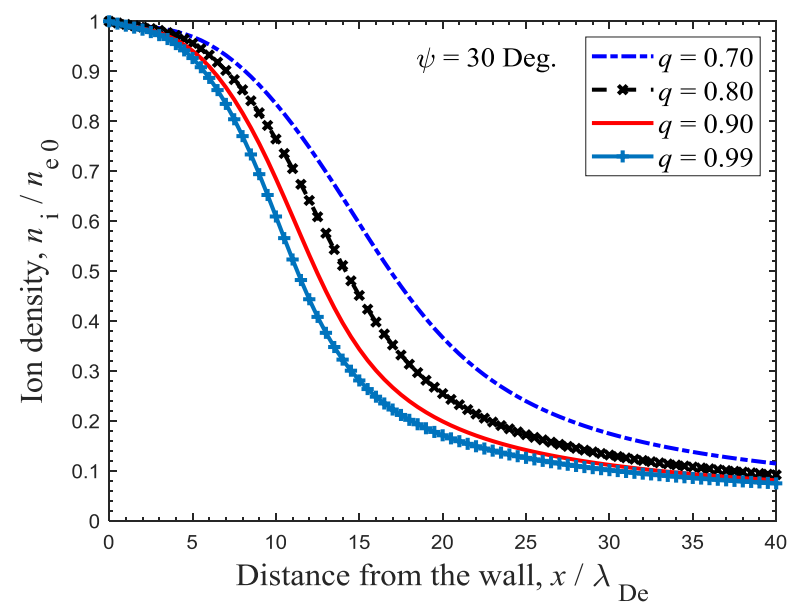

Fig. 3: Ion density profile for different values of nonextensive parameter.

From the figures, it is seen that both ion and electron densities decrease towards the wall for all the four cases of nonextensive parameter $q$. As the nonextensive parameter $q$ increases, the decreasing rate of positive ions and electrons are much faster and attains their minimum at the wall. Moreover, the population of positive ions at the wall decreases with the increase in nonextensive parameter $q$ and the minimum electron density point shifts towards the sheath entrance.

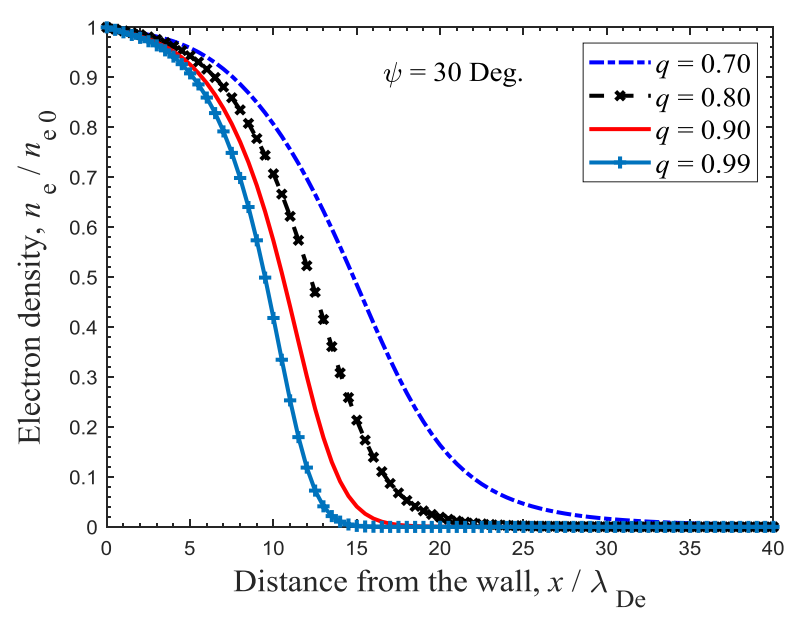

Fig. 4: Electron density profile for different values of nonextensive parameter.

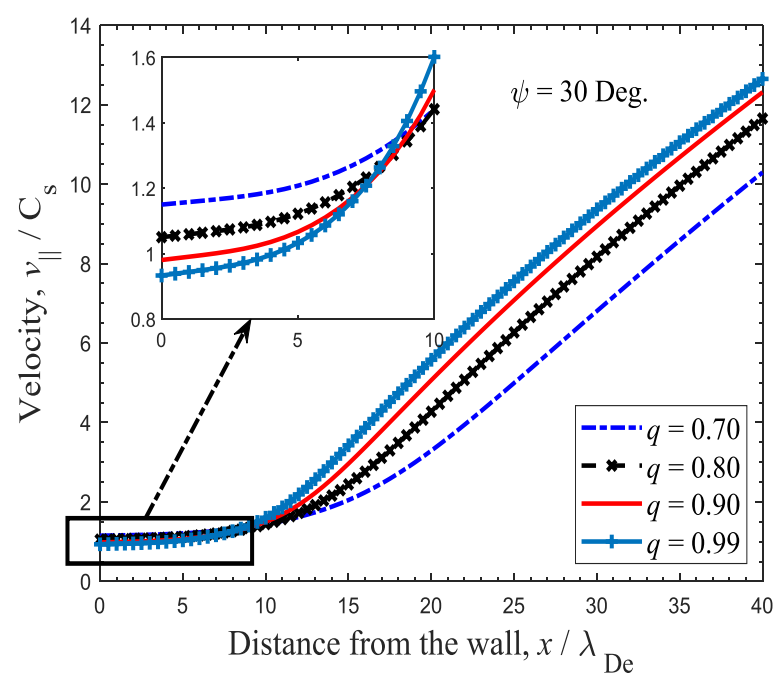

Fig. 5: Variation of parallel component of ion velocity in the sheath region for different values of nonextensive parameter.

The parallel component of ions velocity profile in the plasma-wall transition region for four different values of nonextensive parameter $q$ is shown in Fig. 5. It is seen that the parallel component velocity is almost constant (not significantly increases) up to about 9 electron Debye lengths from the sheath entrance and after that the velocity abruptly increases towards the wall. However, the increment rate of ion's velocity at the wall is higher when the $q$-nonextensive electron distribution becomes 
Boltzmann. As the parameter $q$ increases, the entrance velocity of ions decreases according to equation (19) and hence the velocity decreases at the entrance which is clearly seen in blow up part in Fig. 5. The magnitude of velocity at the wall increases from about $10.32 C_{s}$ to $12.65 C_{s}$ with the increase in nonextensive parameter from 0.70 to 0.99 .

The obliqueness of magnetic field acting on the xzplane determines the strength of magnetic force, which has influence on both the parallel and perpendicular components of ion velocity. The variation of parallel and perpendicular components of ion velocity in the plasma-wall transition region at constant nonextensive parameter $q=0.70$ is shown in Fig. 6. It is found that both the parallel and perpendicular components of ion velocity increase towards the sheath region, although the increment rate of parallel component velocity is higher for small obliqueness of magnetic field. In such cases, the parallel component has dominant contribution on the resultant velocity of positive ions. With the increase in obliqueness of magnetic field, the perpendicular component sharply increases due to increment of drift velocity along the y-direction.

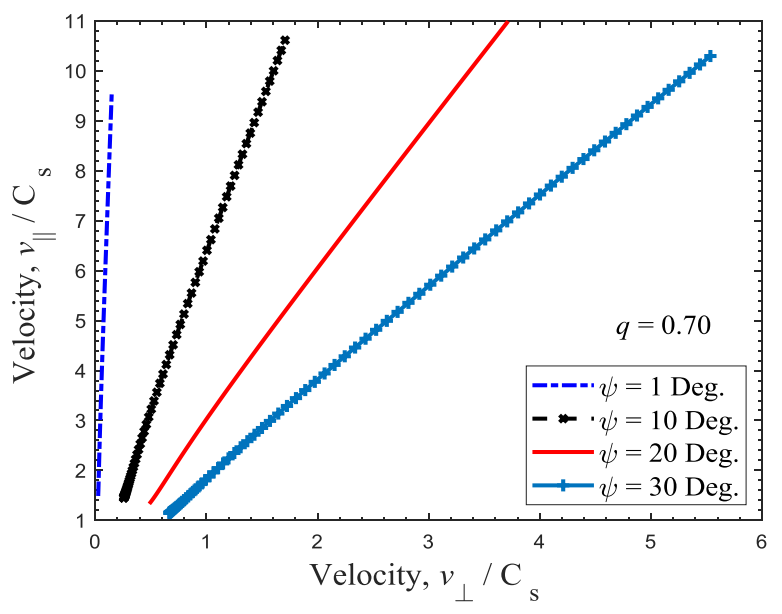

Fig. 6: Parallel and perpendicular components of ion velocity in the sheath region.

The variation of normalized electric potential in the sheath region for four different values of nonextensive parameter $q$ at constant obliqueness of magnetic field 30 Deg. is depicted in Fig. 7. It is seen from Fig. 7 that the magnitude of potential monotonically increases towards the wall for all the cases. Although the potential is not significantly changed near to sheath entrance with nonextensive parameter $q$, the sharpness of potential gets increased towards the wall which indicates that the slope of potential curve increases for the increasing value of nonextensive parameter $q$. As the $q$ increases, the initial flux of ions at the sheath entrance decreases and the flux of ions reaching at the wall also decreases following the conservation of flux and hence the magnitude of wall potential gets increased.

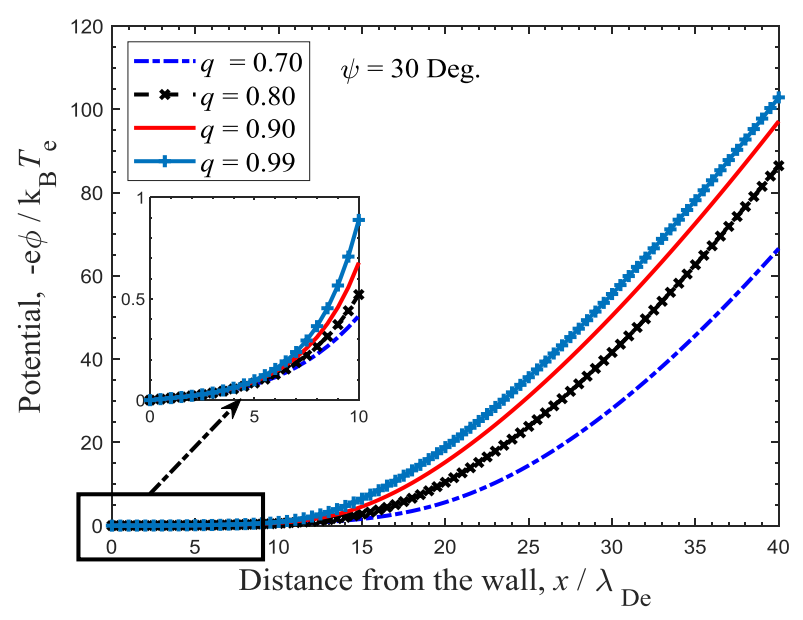

Fig. 7: Electrostatic potential profile for different values of nonextensive parameter.

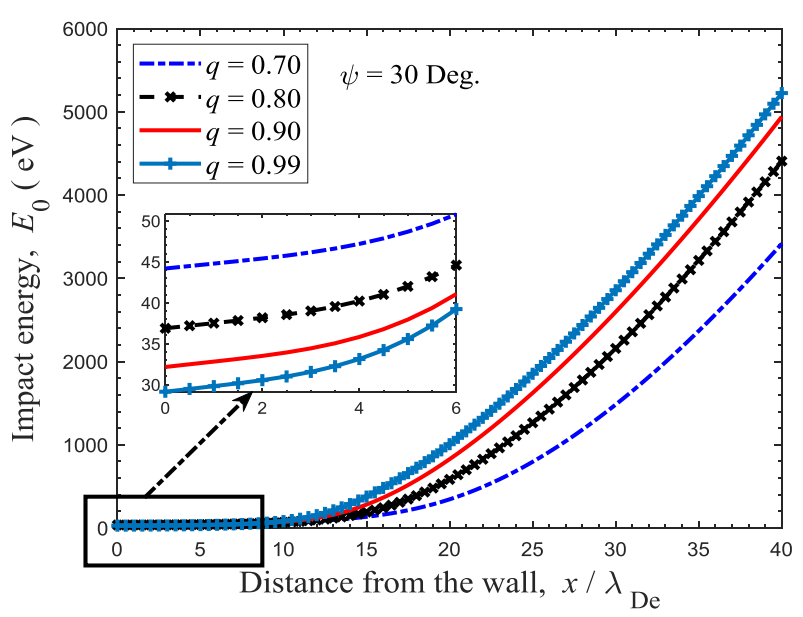

Fig. 8: lons impact energy profile for different values of nonextensive parameter.

Because of negative potential of the wall, the positive ions are accelerated and hence its impact energy also increases which is illustrated in Fig. 8. This impact energy of ions striking the wall determines the sputtering yield, particle reflection and absorption coefficients. The energy flow towards the wall by ions increases with the increase in nonextensive parameter $q$. To study the effect energy flow towards the wall, we present the 
physical sputtering for the tungsten and molybdenum surfaces in Fig. 9. The probability of physical sputtering process is very low below the threshold energy about $220 \mathrm{eV}$ for the $\mathrm{W}$-surface (Fig. 9(a)) and $98 \mathrm{eV}$ for the Mo-surface (Fig. 9(b)) then after it monotonically increases with the increase in impact energy of projectiles. It is found that the physical sputtering yield is low for the Wsurface than that of Mo-surface. Further increment of impact energy of ions, the sputtering rate does not significantly increase after the energy of deuterium ions goes beyond about $1700 \mathrm{eV}$ for Wsurface and $750 \mathrm{eV}$ for Mo-surface. This is because the collision cascades between projectiles and target atoms penetrate deep into the target surface and therefore less energy is transferred to the surface atoms.

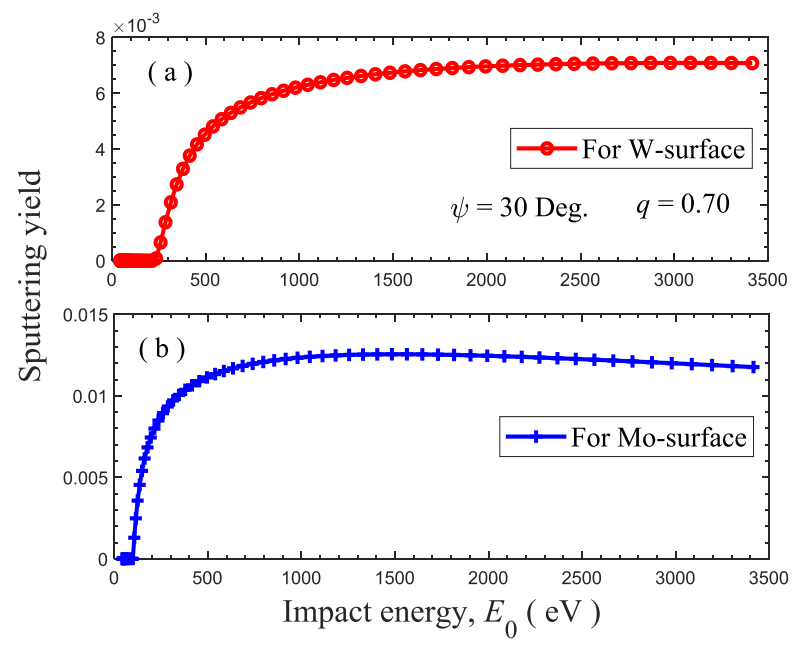

Fig. 9: Sputtering yield as the function ions impact energy for (a) tungsten surface and (b) molybdenum surface.

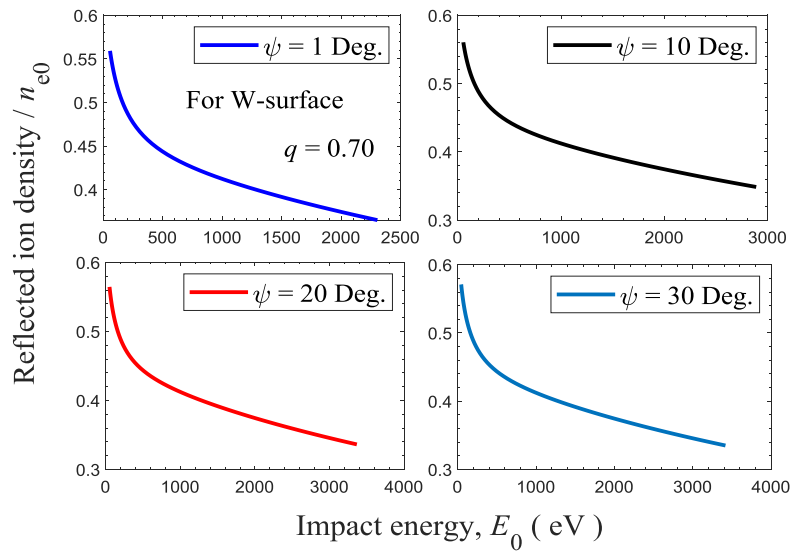

Fig. 10: Reflected ion density form the W-surface.

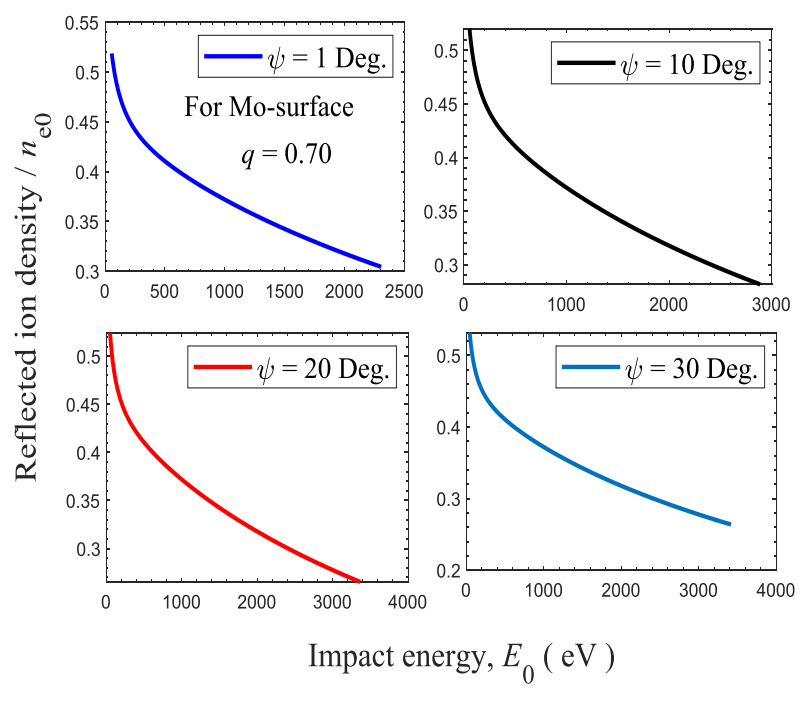

Fig. 11: Reflected ion density form the Mo-surface.

The particle reflection from the target surface is affected by the energy of projectiles and strength of magnetic Lorentz force as well. In order to estimate the reflected density of positive ions (projectiles) from the tungsten and molybdenum surfaces, we present the reflected ion density as the function of impact energy for the different values of obliqueness of magnetic field which are depicted in Figs. 10 and 11, respectively. With the increase in obliqueness of magnetic field from (1-30) Deg., the energy of positive ions striking the wall increases from about $2300 \mathrm{eV}$ to $3400 \mathrm{eV}$. This results in the decrease of reflected ion density from about $0.365 n_{e 0}$ to $0.335 n_{e 0}$ (Fig. 10) and $0.304 n_{e 0}$ to $0.264 n_{e 0}$ (Fig. 11) for tungsten and molybdenum surfaces, respectively.

However, the positive ions in the magnetic field dominant region have higher probability of particle reflection from the PFMs. It is found that the reflected ion density decreases for both the surfaces (tungsten and molybdenum), although the reflection of deuterium ions is higher for tungsten surface than that of molybdenum surface. When the energy of ions reaching the wall increases, the energy transfer due to elastic collision between projectiles and wall is maximum and exceeds threshold energy of target atoms which creates vacant lattice space on the surface of the target. These vacant lattice positions are occupied by the incident ions and hence the reflected ion density falls down. The threshold energy for the tungsten atoms is so higher than that for molybdenum atoms in the target surface. Therefore, the reflected ion density for the $\mathrm{W}$-surface is higher than the Mo-surface. 
The variation of absorbed ion density as the function of impact energy and obliqueness of magnetic field for the tungsten and molybdenum surfaces are shown in Figs. 12 and 13, respectively. It is found that the ion absorption rate increases with the increase in ion's impact energy for both the surfaces, though the absorption is lower for the $\mathrm{W}$-surface than that of Mo-surface. The threshold energy of $\mathrm{W}$-surface is higher than that of Mosurface thereby more energy is required in order to make the interstitial displacement of tungsten atoms from its lattice sites and hence ion absorption coefficient is lower for the $\mathrm{W}$-surface. For instance, when the striking energy of ions at the wall is 2300 $\mathrm{eV}, 0.635 n_{e 0}$ incident ions are absorbed by the $\mathrm{W}$ surface (Fig. 12) whereas the value for Mo-surface is $0.696 n_{e 0}$ (Fig. 13).

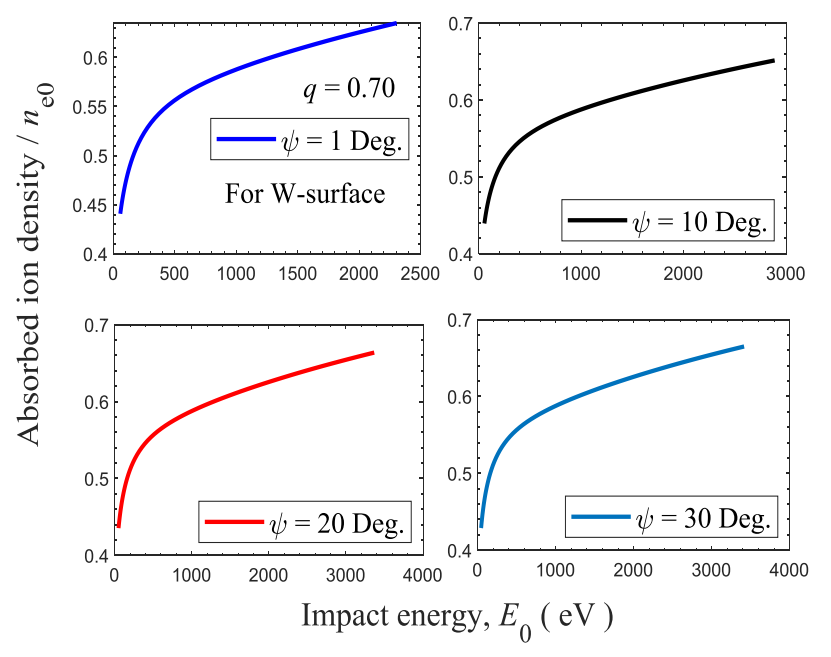

Fig. 12: Absorbed ion density form the W-surface.

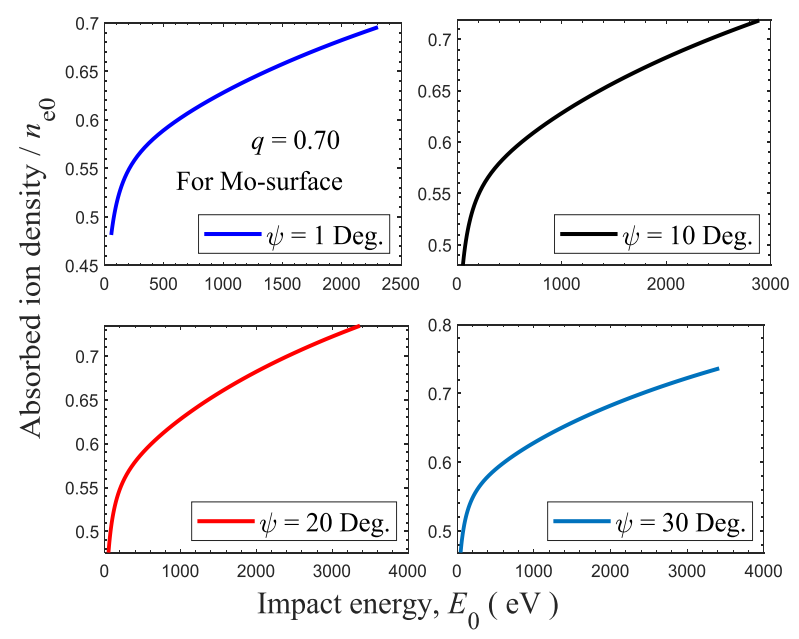

Fig. 13: Absorbed ion density form the Mo-surface.

\section{CONCLUSIONS}

The interaction of magnetized plasma, consisting of warm deuterium ions and $q$-nonextensive distributed electrons, with tungsten and molybdenum surfaces was studied by using fluid theory. The electric fields near the wall are strong enough so it was assumed that the projectiles incident normally. The Bohm's velocity of ions at the sheath entrance was affected by the presence of nonextensive distributed electrons and temperature of positive ions. The particle densities decreased towards the wall and the decreasing rate of electron density is much faster than that of positive ions. The parallel component of velocity abruptly increases after about 9 electron Debye lengths from the sheath entrance. The orientation of magnetic field affected the particles flow along its parallel and perpendicular directions. Although the nature of potential distribution was monotonic function for all choice of nonextensive parameters, the slope of potential function increased towards the wall with the increase in degree of nonextensivity. Due to negative potential developed at the wall, positive ions accelerated and hence its impact energy increased.

The obliqueness of magnetic field and flow of energy determined the physical sputtering, particle reflection and absorption from the target surface. The reflected ion density decreased for both the tungsten and molybdenum surfaces with the increase in impact energy of incident projectiles; however, more particles are reflected back from the $\mathrm{W}$-surface as it has high interstitial displacement energy than Mo-surface. Therefore, the Mo-surface has high absorption coefficient compared to that of $\mathrm{W}$-surface. Furthermore, the present work concluded that reflection coefficient of $\mathrm{W}$-surface is higher than Mo-surface by about (7-19) \% for the incident energy range $40 \mathrm{eV}$ to $3.5 \mathrm{keV}$. This has useful applications in the modelling of plasma facing material wall in future fusion devices, for example, in ITER.

\section{ACKNOWLEDGEMENTS}

Suresh Basnet would like to acknowledge University Grants Commission, Sanothimi, Bhaktapur, Nepal for the Ph.D. fellowship.

\section{REFERENCES}

[1] Riemann, K. U. The Bohm criterion and boundary conditions for a multicomponent system. IEEE Transactions on Plasma Science, 23, 709-716 (1995). 
[2] Valentini, H. B. and Herrmann, F. Boundary value problems for multi-component plasmas and generalized Bohm criterion. Journal of Physics D: Applied Physics, 29, 1175-1180 (1996).

[3] Sato, K., Miyawaki, F., and Fukui, W. Sheath and presheath in a collisionless open - field plasma. Physics of Fluids B: Plasma Physics 1, 725-733 (1989).

[4] Oksuz, L. and Hershkowitz, N. First experimental measurements of the plasma potential throughout the presheath and sheath at a boundary in a weakly collisional plasma. Physical Review Letters, 89, 145001-3 (2002).

[5] Scheiner, B., Baalrud, S. D., Yee, B. T., Hopkins, M. M., and Barnat, E. V. Theory of the electron sheath and presheath. Physics of Plasmas, 22, 123520-13 (2015).

[6] Stangeby, P. C. The plasma boundary of magnetic fusion devices. Institute of Physics Publishing, Bristol and Philadelphia (2000).

[7] Barabash, V., Akiba, M., Mazul, I., Ulrickson, M., and Vieider, G. Selection, development and characterisation of plasma facing materials for ITER. Journal of Nuclear Materials, 233-237, 718-723 (1996).

[8] Yoshida, N. Review of recent works in development and evaluation of high-Z plasma facing materials. Journal of Nuclear Materials, 266-269, 197-206 (1999).

[9] Sheridan, T. E. and Goree, J. A. Analytical expression for the electric potential in the plasma sheath. IEEE Transactions on Plasma Science, 17, 884-888 (1989).

[10] Garcia-Rosales, C. Erosion processes in plasmawall interactions. Journal of Nuclear Materials, 211, 202-214 (1994).

[11] El Kaouini, M., Chatei, H., Driouch, I., and El Hammouti, M. Ion temperature effect on Bohm criterion for magnetized plasma sheath. Journal of Fusion Energy, 30, 199-204 (2011).

[12] Philipps, V. Tungsten as material for plasmafacing components in fusion devices. Journal of Nuclear Materials, 415, S2-S9 (2011).
[13] Cottrell, G. A. Sigma phase formation in irradiated tungsten, tantalum and molybdenum in a fusion power plant. Journal of Nuclear Materials, 334, 166-168 (2004).

[14] Neu, R. High-Z plasma facing components in fusion devices: boundary conditions and operational experiences. Physica Scripta, T123, 33-44 (2006).

[15] Hatami, M. M., Tribeche, M and Mamun A. A. Debye length and electric potential in magnetized nonextensive plasma. Physics of Plasmas, 25, 094502-4 (2018).

[16] El Ghani, O., Driouch, I., and Chatei, H. Effects of non-extensive electrons on the sheath of dusty plasmas with variable dust charge. Contributions to Plasma Physics, 59, e201900030-10 (2019).

[17] Tsallis, C. Possible generalization of BoltzmannGibbs statistics. Journal of Statistical Physics, 52, 479-487 (1988).

[18] Tsallis, C., Mendes, R. and Plastino, A. R. The role of constraints within generalized nonextensive statistics. Physica A, 261, 534-554 (1998).

[19] Thomas, E. W., Janev, R. K., \& Smith, J. Scaling of particle reflection coefficients. Nuclear Instruments and Methods in Physics Research Section B: Beam Interactions with Materials and Atoms, 69, 427-436 (1992).

[20] Gyergyek, T. and Kovacic, J. Fluid model of the sheath in front of a floating electrode immersed in a magnetized plasma with oblique magnetic field: Some comments on ion source terms and ion temperature effects. Physics of Plasmas, 22, 0435021-0435024 (2015).

[21] Liu, Y., Liu, S. Q., and Zhou, L. Bohm criterion in a dusty plasma with nonextensive electrons and cold ions. Physics of Plasmas, 20, 043702-9 (2013).

[22] Eckstein, W. and Preuss, R. New fit formulae for the sputtering yield. Journal of Nuclear Materials, 320, 209-213 (2003). 